\title{
Le Dieu Rao de Mangareva et le Curcuma longa
}

\section{Catherine Orliac}

\section{(2) OpenEdition}

Journals

Édition électronique

URL : http://journals.openedition.org/jso/1535

DOI : $10.4000 /$ jso. 1535

ISSN : 1760-7256

Éditeur

Société des océanistes

Édition imprimée

Date de publication : 1 décembre 2002

Pagination : 201-207

ISSN : 0300-953x

\section{Référence électronique}

Catherine Orliac, "Le Dieu Rao de Mangareva et le Curcuma longa », Journal de la Société des Océanistes [En ligne], 114-115 | Année 2002, mis en ligne le 26 mai 2008, consulté le 22 mars 2021. URL : http:// journals.openedition.org/jso/1535; DOI : https://doi.org/10.4000/jso.1535

Journal de la société des océanistes est mis à disposition selon les termes de la Licence Creative Commons Attribution - Pas d'Utilisation Commerciale - Pas de Modification 4.0 International. 


\title{
Le Dieu Rao de Mangareva et le Curcuma longa
}

par

\author{
Catherine ORLIAC *
}

\section{RÉSUMÉ}

Il y a une vingtaine d'années, Jacques Barrau me fit découvrir une sculpture d'une étonnante modernité : il s'agissait d'une représentation du dieu Rao, invoqué au moment de la plantation du Curcuma longa, à Mangareva, dans l'archipel des Gambier. Lorsqu'il prit sa retraite en 1995, il m'offrit une copie de cette ouvre qu'il affectionnait particulièrement.

Mots-CLÉs : dieux de Mangareva, art des Gambier, Curcuma longa, Thespesia populnea.

Lors de l'une de nos premières rencontres dans les années 1980, Jacques Barrau me fit découvrir une sculpture surprenante des îles Gambier représentant le dieu Rao, invoqué au moment de la plantation du Curcuma longa. Il possédait une copie ${ }^{1}$ de cette œuvre qu'il affectionnait particulièrement. Elle était exposée sur une des étagères proches de son bureau parmi d'autres objets océaniens. Lorsqu'il prit sa retraite en 1995, il m'offrit cette petite sculpture d'une étonnante modernité.

Cette représentation du dieu Rao qu'aimait Jacques Barrau est aujourd'hui exposée au pavillon des Sessions du musée du Louvre (fig.1) ; elle provient des îles Gambier, un petit archipel situé à $1600 \mathrm{~km}$ de Tahiti, par $23^{\circ} 18 \mathrm{de}$

\begin{abstract}
About twenty years ago, Jacques Barrau showed me a strange sculpture with a modern style. It was the image of Rao, a special god invoked during Curcuma longa planting by people of Mangareva, in Gambier archipelago. When he retired in 1995, Jacques Barrau offered me a copie of this piece of art that he loved so much.
\end{abstract}

KeYwords : Mangareva gods, Gambier art, Curcuma longa, Thespesia populnea. latitude Sud, $134^{\circ} 51$ et $135^{\circ} 07$ de longitude Ouest. Cette œuvre, longue de $106 \mathrm{~cm}$ et large de 15,5 cm, a été collectée en 1836 à Mangareva, la plus grande île de l'archipel, par les Pères de la Congrégation des Sacrés-Cœurs de Picpus au moment de la conversion de ses habitants au christianisme ; cette année là, le roi de Mangareva, Te-Maputeoa, fut baptisé et prit le nom de Grégoire Stanislas en hommage au pape Grégoire XVI. L'évangélisation de l'archipel était l'œuvre des Pères Honoré Laval et François d'Assise Caret arrivés sur la Sylphide deux ans plus tôt en compagnie d'un interprète originaire de Nouvelle-Zélande (Buck, 1938 : 99 ; Jacquemin 2000 : 309). Comme ailleurs en Polynésie, cette conversion au christianisme s'accompagna

* Archéologue au CNRS, UMR 7041 à Nanterre, et GDR 1170 au laboratoire d'ethnobiologie-biogéographie du Muséum national d'Histoire naturelle.

1. Cette reproduction en terre cuite, d'une vingtaine de centimètres de hauteur, avait été réalisée par un ami polynésien. Une photo couleur de l'objet du musée du Louvre était fixée au mur, non loin de la statuette. 
de nombreux autodafés, de la destruction des « idoles » et des sanctuaires ancestraux. Cependant, comme le note le Père Mouly (Jacquemin, 2000 : 309) « on eut soin de sauver de l'hécatombe les spécimens les plus remarquables de l'art mangarévien », vibrants témoignages de la victoire des missionnaires sur le paganisme. Le Père Caret avait en effet envoyé à Paris une malle contenant des dieux païens notamment Mainaraughi ou Kotu (dieu à quatre pieds), Minita (idole en corde), Rongo (idole de l'arc-en-ciel), Tupo et Rao « idoles de l'impureté ». D'après les missionnaires, le dieu $R a o$, qui portait le $\mathrm{n}^{\mathrm{o}} 3$, «était consacré (à) une plante appelée ranga dont la fleur est jaune et d'une odeur très pénétrante. Les jeunes gens portaient des tapas colorés en jaune avec cette fleur, durant les jours de débauche » (Jacquemin, $2000: 309$ ).

La plante dont il est question dans l'inventaire de Caret n'est autre que le Curcuma longa L. ${ }^{2}$, une zingibéracée très prisée des Mangaréviens qu'ils nommaient autrefois ranga ou rega; ce nom vernaculaire désignait non seulement la plante elle-même mais également la préparation culinaire issue de la cuisson du tubercule (Buck, $1938: 214)^{3}$.

Le Curcuma longa est une plante herbacée vivace de 0,50 à $1 \mathrm{~m}$ de hauteur (Barrau, 1962: 119), à tubercule dont la chair est d'un jaune orangé ; c'est à la fois une plante tinctoriale, une plante alimentaire et une plante à épice (Barrau, 1963 : 167). (Fig. 3). À Tahiti, son tubercule, qui peut atteindre $15 \mathrm{~cm}$ de long et $2 \mathrm{~cm}$ de diamètre, a une odeur aromatique et une saveur piquante moins prononcée toutefois que celle du gingembre (Pétard,1986: 20-121).

Bien qu'il n'existe plus à l'état sauvage, Curcuma longa, communément nommé safran des Indes ou turmeric, est originaire des Indes (Brown,1931:163 ; Sopher, 1964 : 95 ; Smartt et Simonds, $1995: 493$ ) peut-être du centre indien de Vavilov ${ }^{4}$. Le nom du turmeric en sanskrit, ainsi que dans d'autres langues polynésiennes ${ }^{5}$, signifie la couleur jaune qui est une couleur hautement sacrée aux Indes et en Océanie ${ }^{6}$.

Introduite par l'homme dès le début du peuplement sur les seules îles hautes de Mélanésie, Micronésie et Polynésie, cette plante « figurait parmi les articles qui, aux temps anciens [...] faisait l'objet d'échanges » (Barrau, 1962 : 120). Elle y était très prisée comme source de teinture pour les tapa (étoffes d'écorces battues) et pour les peintures corporelles lors des cérémonies (Brown, 1931 : 163). On l'employait également comme aliment et comme condiment, notamment aux îles Samoa et en Nouvelle-Calédonie ${ }^{7}$. Elle était aussi utilisée en Polynésie dans la préparation de médicaments, à Tahiti en particulier pour soigner les affections gastriques et hépatiques (Pétard, 1986: 121) et pour le traitement des blennorragies et des leucorrhées (Pétard, $1960: 85)^{8}$.

En Asie du Sud-Est et en Océanie, cette plante à croissance rapide a toujours eu des propriétés magiques souvent liées à la fertilité de la terre (Purseglove, 1972 : 523) ; elle était notamment utilisée pour peindre les corps lors de rites associés à la naissance des enfants, au mariage, à la mort et à l'agriculture (Purseglove et al., 1981 : 532. L'usage cérémoniel du Curcuma s'est répandu très tôt en Océanie et son utilisation rituelle en Polynésie trouverait son ancienneté et son origine en Asie du Sud-Est.

En Polynésie, selon l'âge de la plante, la teinture de Curcuma varie du jaune canari, à l'orange, au rouge et au jaune moutarde; on peut aussi obtenir un jaune-chrome de toute beauté ; cette couleur est due à la présence dans le tubercule de $0,5 \%$ de curcumine (Pétard, $1986: 120$ ).

Le colorant était traditionnellement préparé en Polynésie de deux façons différentes : d'après F. Brown, lors de certaines cérémonies marquisiennes, le rhizome était gratté, puis cuit au four jusqu'à ce qu'il soit mou, enfin pressé dans un

2. Curcuma domestica syn C. longa (Purseglove, $1972: 523$ ).

3. Noms vernaculaires du Curcuma en Polynésie orientale d'après J. Barrau (1956: 90), P. Pétard (1986: 120), F. Brown (1931 : 163) et W.A. Whistler (1991:62) :

Cook : renga, Tahiti, Tubuai : rea, Marquises : eka, ena, Tuamotu : talea, Australes : terea, Hawaii : olena, Rapa : lea.

4. Voir J. Barrau (1962: 120). Selon D.E. Sopher (1964: 95), «The plant must have been domesticated somewhere in southern Asia [...] selection and domestication [...] must certainly have preceded the time when it was carried eastward across the Pacific by Polynesians as far as Easter Island $»$.

5. Selon Davies (1984: 224), la couleur jaune en tahitien se dit rengarenga ou rearea, le Curcuma se dit rea (notés respectivement re'are'a et re'a dans la graphie actuelle).

6. En Polynésie les couleurs rouge, rouge-orangé et jaune « was the saced, royal or divine colour » (Sopher, 1964: 113). Selon Marau Taaroa, à Tahiti, « le maro 'ura (la ceinture rouge) était insigne de royauté et le maro tea (le ceinture jaune) insigne du grand prêtre » (Takau Pomare, $1971: 43$ ).

7. Aux Samoa, le résidu du tubercule râpé pour en extraire le colorant était cuit au four mélangé de crème de coco ; le Curcuma était aussi utilisé en Nouvelle-Calédonie sous forme de sauce pour rehausser le goût des féculents (Barrau, 1962 : 120). Il n'était pas utilisé comme condiment en Polynésie (Sopher, 1964 : 114).

8. Il était aussi administré comme tonique et pour les affections ORL à Hawaii (Sopher, 15964 : 118). 
tapa pour en exprimer le jus ; il n'était jamais utilisé par les personnes âgées mais pour peindre le corps de jeunes filles et de jeunes garçons qui étaient tapu (sacrés) durant les danses et les fêtes (Brown, 1931 : 163). Selon P. Pétard (1960 : 84), à Tahiti et aux Marquises, les jeunes gens qui prenaient part aux danses rituelles « devaient se teindre le corps d'une teinture dont la préparation, accompagnée de diverses cérémonies, était réservée à de vieux indigènes, opérant en pleine brousse et loin des villages. Ils râpaient des racines fraîches qu'ils faisaient macérer dans de l'huile de coco [...] il ne restait plus qu'à filtrer [...] et à distribuer le liquide aux danseurs $\gg{ }^{9}$.

Le Curcuma longa n'est plus cultivé de nos jours à Mangareva ${ }^{10}$ mais autrefois, il était si hautement estimé qu'il était placé sous la protection de trois dieux: Meihara-iti, Ragahau et Rogo (Laval, 1938 : 272); Rogo était chargé de faire tomber la pluie et de produire des nourritures; il était symbolisé par l'arc-en-ciel et la brume et possédait les attributs du dieu de l'horticulture (Buck, $1938: 224,422)^{11}$. Deux autres dieux, Rao et Tupo, étaient plus particulièrement invoqués au cours de la plantation du Curcuma (rega $)^{12}$; des prêtres ${ }^{13}$, nommés taura-rega, conduisaient les cérémonies offertes au moment de sa mise en culture et de la cuisson de ses tubercules.

D'après le Père Honoré Laval, la plantation du rega commençait peu de temps après la récolte du fruit de l'arbre à pain, quelques fruits ayant été laissés sur l'arbre pour fournir de la nourriture aux travailleurs. Les racines du Curcuma étaient coupées et plantées comme celles des patates douces (Ipomoea batatas) puis les prêtres concernés se rassemblaient sur le marae (sanctuaire), y plantaient des roseaux (Miscanthus floridulus) avec des banderoles d'écorces de mûrier à papier (Broussonetia papyrifera) et récitaient des prières nommées putoka. Lorsque les pousses perçaient le sol, elles étaient protégées des rats et du vent par des feuilles d'Hibiscus tiliaceus (Laval, 1938 : 273).

La récolte avait lieu l'année suivante, chaque pied ayant en moyenne cinq à huit tubercules. Au moment de la récolte, une grande quantité de nourritures était mise à cuire pour les prêtres et les travailleurs. Les tubercules étaient arrachés et lavés, ce travail étant ponctué de chants appelés kapa en l'honneur du rega. Les tubercules, râpés par les prêtres, étaient ensuite placés dans un grand bol en bois (umete), lavés plusieurs fois, puis disposés dans un récipient appelé aoga percé au fond d'un petit trou. Le Curcuma était ensuite cuit dans un four en terre traditionnel dont les pierres chauffées n'étaient pas recouvertes d'algues mais de feuilles de nono (Morinda citrifolia). Au moment de l'ouverture du four, une prière était faite en l'honneur des dieux du rega, puis le prêtre ouvrait le four et s'il jugeait la cuisson terminée, il retournait le récipient et soufflait dans le trou situé à la base du aoga. Si le Curcuma était bien cuit, il sortait facilement du moule, mais s'il restait coincé sur l'un des côtés du récipient et se brisait, cela signifiait que l'un des travailleurs n'avait pas observé la loi de l'abstinence. Le rega solidifié appartenait à la famille qui l'avait cultivé. Il était consommé à l'exception de la mousse formée à la surface du récipient qui était réservée pour les dieux (Laval, 1938 : 273-274) ${ }^{14}$.

Comme l'indique l'inscription portée par le Père Caret sur la sculpture du dieu Rao du musée du Louvre, le Curcuma longa servait également aux îles Gambier pour teindre les tapa ${ }^{15}$ portés par les jeunes gens lors de certaines cérémonies ; il était aussi utilisé pour les peintures corporelles qui étaient apposées à l'occasion de rituels associés à la naissance d'un enfant, l'accès aux fonctions de prêtre, ou bien encore au moment des funérailles. Le rega était appliqué sur le ventre des futures mères au cours du sixième mois de grossesse par un rogorogo ${ }^{16}$ de haut rang lors d'une cérémonie appelée pani kopu (peinture de l'abdomen); le rogorogo commençait par enduire l'abdomen puis les flancs de la jeune femme, ensuite le dos et jusqu'au cou. Il peignait enfin les membres inférieurs depuis les cuisses jusqu'aux chevilles (Buck, 1938 : 104). De même, lors de la cérémonie préparatoire à la nomination d'un prêtre, les genoux du candidat à la

9. Selon Sopher (1964: 112), cette opération par filtrage était un travail très délicat.

10. Il a cessé d'être cultivé aux Gambier après l'arrivée des missionnaires alors qu'étaient abandonnés les rites ancestraux.

11. Le Père Caret avait également envoyé à Paris l'effigie de Rongo : «idole de l'arc-en-ciel ».

12. Cf. Laval (1938: 272) et Buck (1938: 463). En tahitien, Ra'o signifie « quelque chose planté dans le sol au moyen d'un enchantement » (Davis, $1984: 22$ ).

13. Les prêtres séjournaient au marae de Te Hau-o-te-vehi et observaient la chasteté durant la plantation du rega (Laval, $1938: 272$ ).

14. Au moment de la récolte, de la préparation et de la cuisson des tubercules, des chants étaient entonnés durant la nuit en l'honneur des dieux. Les travailleurs, ainsi que les prêtres, observaient l'abstinence et les prêtres séjournaient dans des maisons érigées en bord de mer et dédiées aux dieux Meihara-iti et Ragahau.

15. Appelé toga à Mangareva.

16. Rogorogo est une classe d'experts, un groupe d'intellectuels appartenant à l'aristocratie qui est responsable des chants sacrés lors des cérémonies importantes (Buck, 1938 : 304). 
prêtrise étaient recouverts de rega puis frappés avec une feuille de $t i$ (Cordyline fruticosa) ou de canne à sucre (Saccharum officinarum) par le chef des rogorogo (Buck, 1938: 43). Enfin, Caillot remarque que les offrandes déposées près des morts comportaient un paquet de cordes de fibres de coco, un morceau de tapa fait de l'écorce de l'arbre à pain, quelques bols en bois contenant du fruit fermenté de l'arbre à pain et une calebasse avec de l'eau mélangée à du Curcuma. La tradition orale rapporte que la nourriture et le mélange d'eau et de rega devaient alimenter le mort au cours de son voyage post mortem (Caillot, 1910 : 152).

Les textes ethnohistoriques ne livrent pas d'information sur les bois d'œuvre qu'utilisaient les Mangaréviens pour façonner les effigies du dieu Rao. Cependant, quelques fins prélèvements effectués sur la sculpture expédiée par Caret ont permis de découvrir que cette représentation du dieu avait été sculptée dans une branche de Thespesia populnea (Orliac, 2000 : 30). (Fig. 2). Cet arbre, très commun en Polynésie et qui pousse sur le littoral des atolls et des îles hautes, peut atteindre une quinzaine de mètres de hauteur ; appelé miro aux îles Gambier, il poussait autrefois abondamment dans l'archipel.

Le Thespesia populnea jouait un rôle important dans la culture polynésienne traditionnelle. À Tahiti, il était planté dans l'enceinte des marae (sanctuaires) où il était indispensable en tant qu'émanation du dieu Roro'o qui inspirait les prêtres dans leurs dévotions (Henry, 1968 : 394) ; parmi les arbres des marae, il était « le plus sacré [...] celui qui sanctifie » (Henry, $1968:$ 158). Le bois, mais aussi les feuilles et les branches du Thespesia, étaient utilisés lors de nombreuses cérémonies qui se déroulaient sur les lieux de culte. Aux îles Gambier, des baguettes de miro, ornées de banderoles de tapa, étaient employées lors de la cérémonie du eketea au moment de l'initiation d'un prêtre de haut rang (Buck, $1938: 450$ ).

Le Thespesia populnea présente certains points communs avec le Curcuma longa; comme lui, il donne des fleurs jaunes au parfum discret ; de plus ses fruits, ses feuilles et son écorce fournissent un colorant jaune. Le pédoncule du fruit vert laisse en effet exsuder un suc glutineux jaune et le péricarpe du fruit une sève jaune inodore que les Hawaïens employaient pour colorer leur tapa. Anciennement aux îles Marquises, le suc de l'écorce du miro servait à teindre les tapa destinés aux nouveaux nés (Petard, 1986 :120, 218).

En Polynésie, le bois du Thespesia populnea était très recherché par les sculpteurs en raison de ses caractéristiques uniques; en effet, plus connu sous le nom de " bois de rose d'Océanie », il présente une couleur légèrement rosée; de plus, lorsqu'il est travaillé vert, il dégage une odeur poivrée qui disparaît avec le temps; c'est un matériau au grain fin, qui se travaille bien et se polit facilement. Il n'est pas surprenant que le dieu Rao soit sculpté dans une branche de miro car ce bois est souvent cité dans les textes ethnographiques et la tradition orale polynésienne pour la confection des « idoles » et la sculpture d'objets de prestige tels que le siège et le sceptre royal ${ }^{17}$. Il servait également au façonnage des hampes de harpons, des manches d'outils ${ }^{18}$, des traverses de pirogues à balancier, des pagaies ( $c f$. Pétard, 1986: 218) et dans un contexte plus sacré, pour la fabrication des arcs ${ }^{19}$. À Mangareva, le turuturu des chefs, sorte de sceptre élargi et aplati à l'une de ses extrémités, était taillé dans une branche de Thespesia populnea; cet objet était finement poli (Buck, 1938 : 175) ${ }^{20}$.

Cette représentation du dieu Rao, qui est sans conteste une des œuvres « les plus remarquables de l'art océanien » (Jacquemin, $2000: 311$ ), a été datée ${ }^{21}$ par G. Bonani ${ }^{22}$ de AD 793-995, c'està-dire entre le début du $\mathrm{IX}^{\mathrm{e}}$ et le début du XI $\mathrm{XI}^{\mathrm{e}}$ siècle de notre ère. L'ancienneté de cette œuvre paraît surprenante; en effet, en région tropicale et en présence d'insectes xylophages, comment expliquer que ce bois, de dureté moyenne, ait pu se conserver pendant plus de huit cents ans sans subir la moindre dégradation... Ce serait l'objet polynésien le plus ancien que l'on connaisse.

Le dieu Rao, ainsi que d'autres effigies de divinités, étaient conservées dans des édifices

17. ainsi que des petits récipients (umete) finement sculptés comme celui du musée de l'Homme (inventaire ${ }^{\circ}$ 35-61-231) ; cf. Orliac, $1990: 38$.

18. Le manche d'herminette du constructeur de pirogue était toujours fixée «à un manche solide de miro sacré » Henry T. (1968 : 154). Deux manches d'herminettes des collections du Musée de l'Homme (inventaire $n^{\circ} X$ $43-90$ et $\left.n^{\circ} 11-2-1\right)$ sont taillés dans ce bois.

19. En témoigne l'identification d'un objet de ce type conservé dans les collections du musée de l'Homme (inventaire $\mathrm{n}^{\circ}$ 34-33-25) ; $c f$. Orliac, $1990: 38$.

20. Selon Huguenin (1974:467) le « Thespesia populnea : bois de rose 'miro' était autrefois employé pour la sculpture » à Mangareva.

21. À noter que c'est le bois qui est daté, non l'œuvre elle-même. Aucune étude n'a été faite sur l'âge moyen des arbres de ces régions ; J. Florence a observé aux îles Marquises un Thespesia populnea très vieux qui pouvait être centenaire ; il s'agit d'un spécimen rare.

22. Institute of Particles Physics, École polytechnique fédérale de Zurich. Datation calibrée à 2 sigma. 
particuliers que Dumont D’Urville qualifie de « temples des idoles» (Fig. 4). Dans l'enceinte du sanctuaire et à proximité du dieu, étaient érigés des piliers d'offrandes appelés 'ata. Selon F.W. Beechey, ces poteaux avaient la particularité d'être surmontés de « 3 bras gravés, dans les mains desquelles étaient suspendues des offrandes telles des noix de coco sculptées, des bambous, peut-être des instruments de musique... » (Beechey, in Buck,1938 : 456). Un objet de ce type se trouve à Saint-Pétersbourg et un autre au musée de l'Homme; ce dernier a été rapporté de Mangareva en 1884 par le docteur Couteaud, médecin de la Marine nationale. Il mesure 1,80 m de hauteur et $24 \mathrm{~cm}$ de largeur et se compose d'un fût, renflé à mi hauteur pour empêcher l'accès des rats aux nourritures, et d'un plateau circulaire portant quatre bras, ornés de tatouages géométriques, terminés par des mains (Orliac, 1986 : 4-5). Des prélèvements effectués à la base de l'objet et dans sa partie renflée ont permis de constater que les Mangaréviens avaient utilisé du Thespesia populnea pour le façonnage de ce pilier, comme ils l'avaient fait pour sculpter l'image du dieu Rao.

Seule une dizaine d'objets des Gambier, poteaux sculptés ou effigies de divinités, sont aujourd'hui conservés dans quelques rares musées en Italie, en Angleterre et en France. Le musée de La Rochelle possède une de ces sculptures d'une facture très différente de celle du dieu Rao. Collecté en 1838 par Dumont D'Urville, et enregistré dans les collections du musée naval du Louvre sous l'appellation « idole en bois (personnage nu) par l'Astrolabe », cet objet de 108 $\mathrm{cm}$ de hauteur est la représentation d'un dieu inconnu. L'identification du bois de cette œuvre montre qu'elle est également sculptée dans une branche de Thespesia populnea (Orliac, 2002: 3). Il est probable qu'à Mangareva, comme partout ailleurs en Polynésie, le bois de miro était très prisé des sculpteurs, à la fois pour ses qualités techniques et pour sa valeur symbolique.

La petite sculpture en terre cuite du dieu Rao que Jacques Barrau m'a offerte éveille aujourd'hui en moi une foule de souvenirs: elle m'évoque un homme érudit et bienveillant qui marqua profondément ma vie de chercheur, une plante « magique » qu'il connaissait bien, et une société polynésienne originale à jamais disparue.

\section{ANNEXE}

Noms de plantes cités :

Curcuma longa L (Zingiberacées)

Ipomoea batatas (L.) Lamarck (Convolvulacées)

Miscanthus floridulus (Labill.) Warb.ex Schum. et Laut. (Poacées)

Broussonetia papyrifera (L.) Vent. (Moracées)

Hibiscus tiliaceus L. (Malvacées)

Morinda citrifolia L. (Rubiacées)

Cordyline fruticosa (L.) A. Chev. (Agavacées)

Saccharum officinarum L. (Poacées)

Thespesia populnea (L.) Soland. ex Correa (Malvacées)

\section{REMERCIEMENTS}

Je remercie Jacques Florence, botaniste à l'IRD, qui a relu et corrigé cet article ainsi que Michel et Maeva Orliac pour la réalisation graphique de l'illustration.

\section{RÉFÉRENCES}

BArrau J., 1956. L'agriculture vivrière des Polynésiens et des Mélanésiens. Commission du Pacifique Sud. Nouméa.

_, 1962. Les plantes alimentaires de l'Océanie : Origines, distribution et usages. Annales du Musée colonial de Marseille. Faculté des Sciences de Marseille.

_ , 1963. Agriculture des îles Wallis et Futuna. Paris, Journal de la Société des Océanistes 19, pp. 157-172.

Brown F., 1931. Flora of the southeastern Polynesia, t 1 : Monocotyledons. Honolulu, Hawaii., Bernice P. Bishop Museum Bulletin $\mathrm{n}^{\circ} 84$.

Buck P. H., 1938. Ethnology of Mangareva. Honolulu, Hawaii, Bernice. P. Bishop Museum Bulletin $\mathrm{n}^{\mathrm{o}}$ 157.

CaIllot A.C.E., 1910. Histoire de la Polynésie Orientale, Paris, Éd. Leroux.

DAVIES J., 1984. A Tahitian and English dictionary, with introductory remarks on the Polynesian language and a short grammar of the Tahitian dialect. Papeete, Ed. Haere Po No Tahiti.

Henry T., 1968. Tahiti aux temps anciens. Paris, Publications de la Société des Océanistes $n^{\circ} 1$.

Huguenin B., 1974. La végétation des îles Gambier, relevé botanique des espèces introduites. Paris, Fondation Singer Polignac, Cahiers du Pacifique n⿳018, t 2, pp. 459-471.

JACQUEMIN S., 2000. Statue du dieu Rao. Catalogue. Sculptures: Afrique, Asie, Océanie, Amériques. Paris, Réunion des musées Nationaux, Musée du Louvre. Pavillon des Sessions, pp. 309-311. 
Laval H., 1938. Mangareva: Histoire ancienne d'un peuple polynésien, Maison des Pères des SacrésCœurs, Braine-le-Comte, Paris, Geuthner.

OrliaC C., 1986. Identification botanique d'un pilier d'offrande de Mangareva et d'un poteau de case marquisienne. Collection du Musée de l'homme. Rapport multigraphié, CNRS-URA 275.

— 1990. Des arbres et des Dieux, choix des matériaux de sculpture en Polynésie, Paris, Journal de la Société des Océanistes 90-1, pp. 35-42.

— 2000. Des bois et des dieux en Polynésie centrale et orientale : de l'intérêt des études xylologiques pour la connaissance des sculptures polynésiennes. Paris, TECHNE $\mathrm{n}^{\circ} 11$, pp. 27-32 (Laboratoire de recherche des musées de France, CNRS-UMR 171).

—, 2002. Détermination botanique du bois d'œuvre d'une sculpture de Mangareva. Musée de La Rochelle. Rapport multigraphié, CNRS-GDR 1170.

Petard P., 1960. Quelques plantes utiles de Polynésie française, Pteridophytes et Monocotylédones. Thèse de la faculté de médecine et de pharmacie de Marseille.

_, 1986. Plantes utiles de Polynésie, Raau Tahiti. Papeete, Éd. Haere Po No Tahiti.
Purseglove J.W., 1972. Tropical crops. Monocotyledons 2. London, Longman ed.

Purseglove J.W., Brown E.G., Green C.L., Robbins S.R.J., 1981. Turmeric, in Longman ed., Spices, vol. 2, pp. 533-580. London and New York, Tropical Agriculture Series.

Smartt J., Simmonds N.W., 1995. Evolution of crop plants. ( $2^{\text {nd }}$ edition), Essex, Longman Scientific and Technical.

SOPHER D. E., 1964. Indigenous uses of Turmeric (Curcuma domestica) in Asia and Oceania. Anthropos 59, pp. 93-127.

TAKAu Pomare, 1971. Mémoire de Marau Taaroa, dernière reine de Tahiti. Paris, Publication de la Société des Océanistes 27.

Whistler W.A, 1991. Polynesian plant introduction in Islands, plants and Polynesians, an introduction to Polynesian ethnobotany. Proceedings of a symposium sponsored by the Institute of Polynesian Studies, Brigham Young University-Hawaii Campus Lanai, Hawaii. Portland, Oregon, Cox P.A. and Banack S.A. ed., Dioscorides press. 


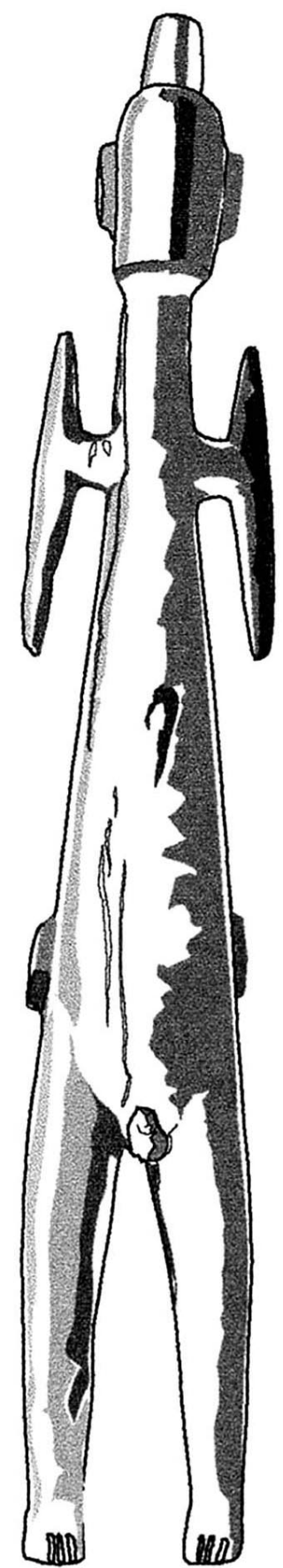

FIG. 1

FIG. 4

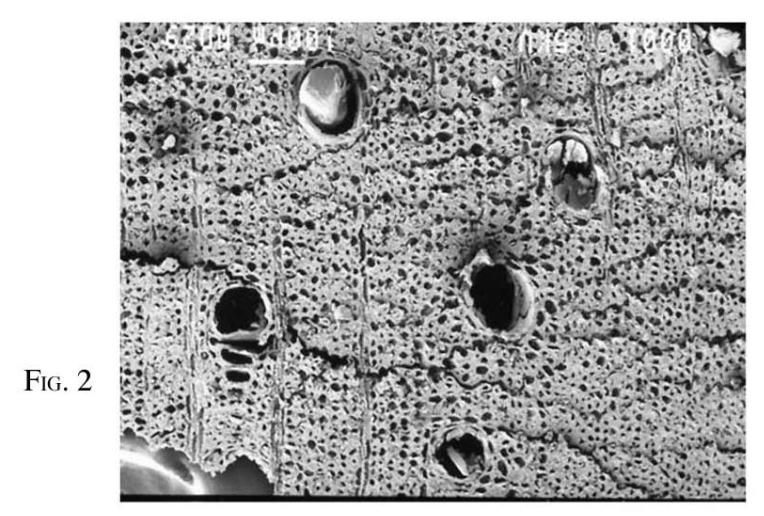

FIG. 3
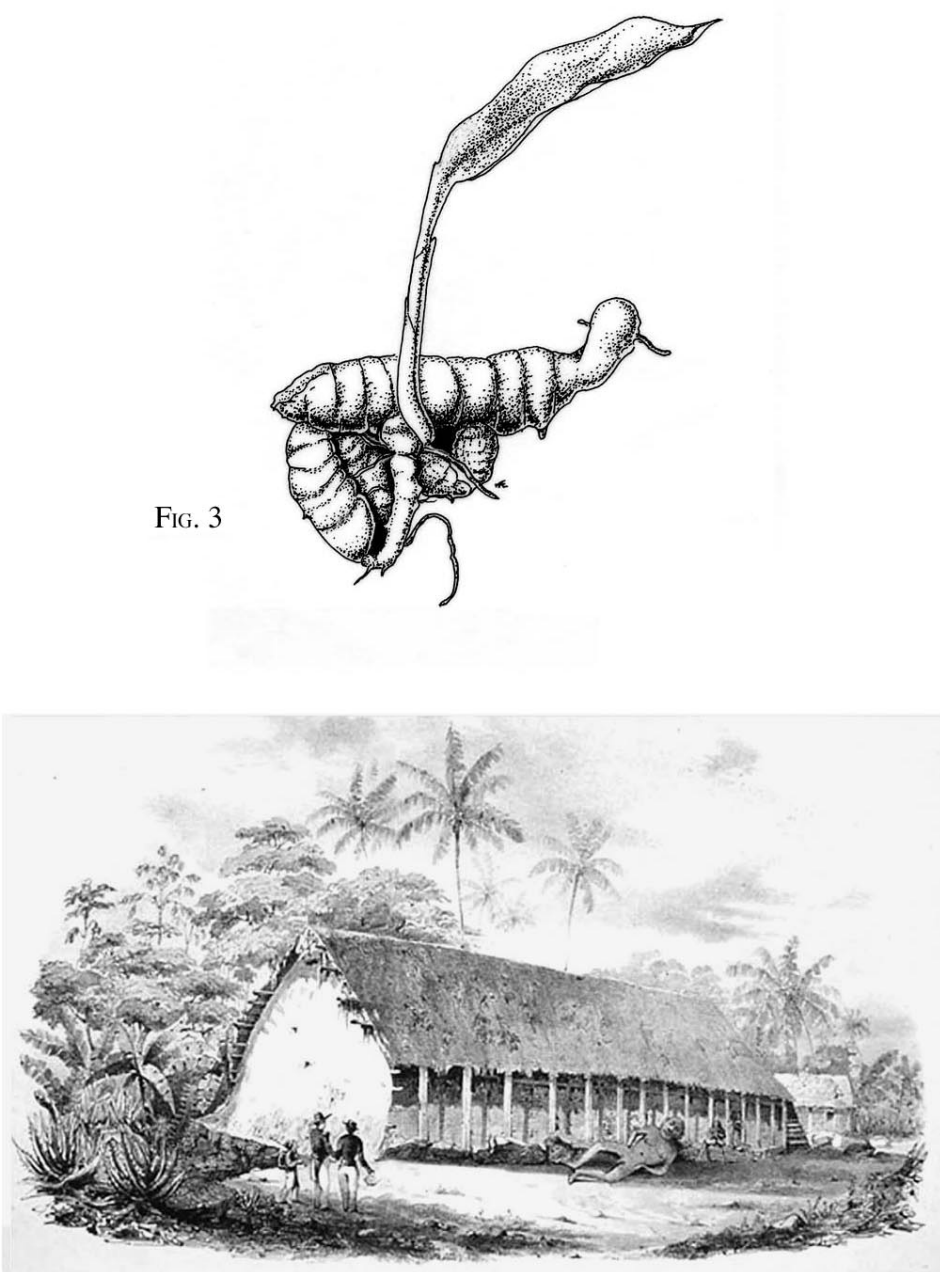

Fig. 1. - Le dieu Rao de Mangareva (Pavillon des Sessions du musée du Louvre ; dessin Michel Orliac).

FIG. 2. - Le dieu Rao : bois de Thespesia populnea (cliché G. Fleck, MHNN).

FIG. 3. - Curcuma longa L. (dessin Maeva Orliac).

FIG. 4. - «Ancien temple des idoles à Manga Reva ». Atlas pittoresque de Dumont D’Urville. 